\title{
GENERAL RECURSIVE FUNCTIONS
}

\author{
JULIA ROBINSON
}

1. Introduction. A primitive recursive function is one which can be obtained from the initial functions $I_{n k}(1 \leqq k \leqq n), O_{n}(n \geqq 0)$, and $S$, by repeated substitution and recursion. Here

$$
I_{n k}\left(x_{1}, \cdots, x_{n}\right)=x_{k}, \quad O_{n}\left(x_{1}, \cdots, x_{n}\right)=0,
$$

$S$ denotes the successor function, and the recursion scheme has the form

$$
F(\mathfrak{x}, 0)=A \mathfrak{x}, \quad F(\mathfrak{x}, S y)=B(\mathfrak{x}, y, F(\mathfrak{x}, y)),
$$

where we have put $\mathfrak{r}=\left(x_{1}, \cdots, x_{n}\right), n \geqq 0 .^{1}$

The class of general recursive functions is obtained if we allow one additional scheme for defining functions, namely

$$
F \mathfrak{x}=\mu y\{A(\mathfrak{x}, y)=0\},
$$

where the symbol on the right denotes the smallest $y$ such that $A(\mathfrak{x}, y)=0$, under the assumption that there is such a $y$ for each $\mathfrak{x}$.

Kleene showed that this definition of general recursive function is equivalent to Herbrand-Gödel metamathematical definition. ${ }^{2}$ In this paper we shall be concerned with the mathematical (as opposed to metamathematical) aspects of the theory of general recursive functions. Starting from the definition stated, we shall investigate the possible restrictions on the defining schemes. Part of the results obtained are already known from the work of Kleene, but we go further in this direction than Kleene did. No previous knowledge of general recursive functions is assumed in this paper.

It will be convenient to have a logical symbolism to express the conditions that appear in applications of the $\mu$-rule. We shall use: $\wedge$ (for every), $\vee$ (there exists), $\wedge$ (and), $\vee$ (or), $\sim$ (not), $\rightarrow$ (if then), $\leftrightarrow$ (if and only if).

The following equivalences will be useful: 1949.

Presented to the Society, November 27, 1948; received by the editors August 22,

${ }^{1}$ Further discussion of this definition may be found, for example, in R. M. Robinson, Primitive recursive functions, Bull. Amer. Math. Soc. vol. 53 (1947) pp. 925-942. This paper will be referred to as PRF. The notation of the present paper is the same as in PRF.

2 See S. C. Kleene, General recursive functions of natural numbers, Math. Ann. vol. 112 (1936) pp. 727-766 and Recursive predicates and quantifiers, Trans. Amer. Math. Soc. vol. 53 (1943) pp. 41-73. 


$$
\begin{gathered}
a=0 \wedge b=0 \leftrightarrow a+b=0, \quad a=0 \vee b=0 \leftrightarrow a b=0, \\
a \neq 0 \leftrightarrow 0^{a}=0 .
\end{gathered}
$$

In connection with the last formula, we recall that $0^{\circ}=1$. Notice also that

$$
\begin{aligned}
& a=b \leftrightarrow(a-b)+(b-a)=0, \\
& a \geqq b \leftrightarrow[(a-b)+b]-a=0
\end{aligned}
$$

where an arbitrary difference function $x-y$ is used; that is, $x-y$ has the usual meaning when $x \geqq y$, but a completely arbitrary meaning for $x<y$.

At various places, we shall need pairing functions, that is functions $J, K$, and $L$ such that

$$
K J(x, y)=x \text { and } L J(x, y)=y .
$$

It will also be convenient to have a one-to-one correspondence between pairs of numbers $(x, y)$ and numbers $z$. This is expressed by the identity

$$
J(K z, L z)=z .
$$

Furthermore, sometimes we shall want $J(x, y)$ to be a monotone increasing function of $x$ and $y$. All of these conditions are satisfied by Cantor's diagonal enumeration. This one-to-one correspondence between pairs of numbers $(x, y)$ and numbers $z$ is established by the equation

$$
2 z=(x+y)^{2}+3 x+y \text {. }
$$

Notice that

$$
8 z+1=(2 x+2 y+1)^{2}+8 x
$$

and hence $\left[(8 z+1)^{1 / 2}\right]$ is either $2 x+2 y+1$ or $2 x+2 y+2$. It follows easily that $J, \dot{K}, L$ can all be obtained by substitution alone, if we adjoin

$$
x^{2}, \quad x+y, \quad x-y, \quad[x / 2], \quad\left[x^{1 / 2}\right]
$$

to the initial functions.

2. Various forms of the $\mu$-rule. A more general form of the $\mu$-rule is

$$
F \mathfrak{x}=\mu y\left\{A_{1}(\mathfrak{x}, y)=A_{2}(\mathfrak{x}, y)\right\} .
$$

This can be reduced to the standard form

$$
F \mathfrak{x}=\mu y\{A(\mathfrak{x}, y)=0\}
$$


by putting $A(\mathfrak{x}, y)=\left|A_{1}(\mathfrak{x}, y)-A_{2}(\mathfrak{x}, y)\right|$. Consider next the more special form

$$
F \mathfrak{x}=\iota y\{B(\mathfrak{x}, y)=0\}
$$

where the right side denotes the only $y$ such that $B(\mathfrak{x}, y)=0$, under the assumption that there is just one such $y$ for each $\mathfrak{x}$. This is readily seen to be equivalent to the preceding form if we take

$$
B(\mathfrak{x}, y)=A(\mathfrak{x}, y)+\sum_{z<y} 0^{A(\mathfrak{x}, z)} .
$$

Indeed, $B(\mathfrak{x}, y)=0$ is equivalent to $A(\mathfrak{x}, y)=0$ and $A(\mathfrak{x}, z) \neq 0$ for $z<y$. Notice that $B(\mathfrak{x}, y)$ is primitive recursive if $A(\mathfrak{x}, y)$ is.

By successive applications of pairing functions, we can reduce the number of components of $\mathfrak{x}=\left(x_{1}, \cdots, x_{n}\right)$ which occur in applications of the $\mu$-rule to one. Consider for example the reduction from two components to one. We are given

$$
F\left(x_{1}, x_{2}\right)=\mu y\left\{A\left(x_{1}, x_{2}, y\right)=0\right\} .
$$

Putting

$$
B(x, y)=A(K x, L x, y)
$$

and

$$
G x=\mu y\{B(x, y)=0\},
$$

we see that

$$
F\left(x_{1}, x_{2}\right)=G J\left(x_{1}, x_{2}\right) .
$$

The same reduction is also valid with $\iota$ in place of $\mu$.

We shall next discuss the relation between the following two forms of the $\mu$-rule:

$$
\begin{aligned}
& F x=\mu y\{A(x, y)=0\}, \\
& G x=\mu z\{B z=x\} .
\end{aligned}
$$

It is easily seen that

$$
F x=L \mu z\left\{A(K z, L z)=0 \wedge K_{z}=x\right\},
$$

where $J, K$, and $L$ are Cantor's pairing functions. Indeed, the equation $z=J(x, y)$, with $x$ fixed, establishes a one-to-one correspondence between values of $y$ satisfying $A(x, y)=0$ and values of $z$ such that $A(K z, L z)=0 \wedge K z=x$. Also, the smallest $y$ corresponds to the smallest $z$. It follows that 


$$
L \mu z\left\{0^{\wedge}\left(K_{z}, L_{z}\right) K z=x\right\}= \begin{cases}F x & \text { if } x \neq 0, \\ 0 & \text { if } x=0 .\end{cases}
$$

Thus, putting

$$
B z=0^{A(K z, L z)} K z
$$

and defining $G x$ as above, we see that

$$
F x=L G x+0^{x} F 0 .
$$

We may also put $F x=H G x$, where

$$
H y=\left\{\begin{array}{lll}
L y & \text { if } & y \neq G 0, \\
F 0 & \text { if } & y=G 0 .
\end{array}\right.
$$

( $H$ is a primitive recursive function.)

The situation is somewhat different for the $\iota$-rule. A more elaborate method is needed for reducing

$$
F x=\iota y\{A(x, y)=0\}
$$

to the use of

$$
G x=\iota z\{B z=x\}
$$

and certain elementary substitutions. As before, we have

$$
F x=L \imath\{A(K z, L z)=0 \wedge K z=x\}
$$

or putting

$$
C z=\operatorname{sgn} A(K z, L z),
$$

also

$$
F x=L \imath z C z=0 \wedge K z=x\} .
$$

For each $x$ there is a unique $z$ such that $K z=x$ and $C z=0$. It is these values of $z$ in which we are interested. We wish to construct a function $B$ from which these values can be read in a simple way, but also so that $\iota z\{B z=x\}$ is a legitimate operation, that is, so that for every $x$ there is exactly one $z$ with $B z=x$, even though the value of $z$ may be immaterial for our purposes.

We shall construct a function $B$ such that

$$
B(2 z)=2 x \leftrightarrow C z=0 \wedge K z=x .
$$

The function $B$ will assume all distinct odd values for odd arguments and for even arguments $2 z$ for which $C z=1$. This will insure the legitimacy of the operation $\iota z\{B z=x\}$. Such a function $B$ may be de- 
fined as follows:

$$
\begin{aligned}
B(2 z) & = \begin{cases}2 K z & \text { if } C z=0, \\
2 z-1+2 \sum_{u \leq z} C u & \text { if } C z=1,\end{cases} \\
B(2 z+1) & =2 z+1+2 \sum_{u \leq z} C u .
\end{aligned}
$$

Notice that $B$ can be obtained from $A$ by substitutions and primitive recursions, and hence is primitive recursive if $A$ is. Finally, we see that if $G$ is defined as above, we have

$$
F x=L[G(2 x) / 2] .
$$

REMARK. Thus, if substitution and primitive recursion are available, the strongest and weakest forms of the $\mu$-rule are equivalent.

3. Elimination of recursion. In this section we shall show that all general recursive functions can be obtained without the use of the recursion scheme, that is, by using only substitution and the $\mu$-rule, if we adjoin suitable functions to the initial functions. Indeed, we shall see that it is sufficient to adjoin $x+y$ and $Q$ if we use the $\mu$-rule in the form $F \mathfrak{x}=\mu y\{A(\mathfrak{x}, y)=0\},{ }^{3}$ and to adjoin $x+y$ and $E$ if we use the more special form of the $\mu$-rule $F x=\mu y\{A y=x\}$. Here $E x=x-\left[x^{1 / 2}\right]^{2}$ is the excess of $x$ over a square, and $Q x=0^{E x}$ is the characteristic function of squares. In the latter case, in place of $E$ we may use either $K$ or $L$. I do not know whether the recursion scheme can be omitted if the $\iota$-rule is substituted for the $\mu$-rule and a finite number of primitive recursive functions are adjoined to the initial functions.

We first recall a device due to Gödel.4 Any finite sequence of numbers $a_{0}, a_{1}, a_{2}, \cdots, a_{y}$ can be obtained as remainders when a fixed number is divided by $1+v(z+1)$, for $z=0,1,2, \cdots, y$. Denoting by $R(u, t)$ the remainder when $u$ is divided by $t$, the required conditions are

$$
R(u, 1+v(z+1))=a_{z} \quad \text { for } 0 \leqq z \leqq y .
$$

These conditions are equivalent to

${ }^{3}$ A similar result was proved by Kleene, adjoining the functions $x+y, x y$, and $0|x-y|$. The method of eliminating recursion is essentially the same as in the present paper. See Kleene, $A$ note on recursive functions, Bull. Amer. Math. Soc. vol. 42 (1936) pp. 544-546.

' See Kurt Gödel, Über formal unentscheidbare Sätze der Principia Mathematica und verwandter Systeme I, Monatshefte für Mathematik und Physik vol. 38 (1931) pp. 173-198. 


$$
u \equiv a_{z}(\bmod 1+v(z+1)), \quad a_{z}<1+v(z+1) .
$$

We need only to choose $v$ sufficiently large and so that the moduli are relatively prime, and then choose $u$ satisfying the congruences.

If we put $w=J(u, v)$, then the sequence is determined from a single number $w$. Putting for brevity

$$
T_{z} w=R(K w, 1+L w \cdot S z),
$$

we have

$$
a_{z}=T_{z} w \quad \text { for } z=0,1,2, \cdots y .
$$

Thus $T_{z} w$ denotes the $z$ th term in the sequence determined by $w$. (Here $J$ can be taken to be any pairing function with suitably associated functions $K$ and $L$.)

We are now ready to consider the elimination of recursive definitions. Suppose that $F$ is defined recursively from $A$ and $B$ by the equations

$$
F(\mathfrak{x}, 0)=A \mathfrak{x}, \quad F(\mathfrak{x}, S y)=B(\mathfrak{x}, y, F(\mathfrak{x}, y)) .
$$

The sequence of numbers

$$
F(\mathfrak{x}, 0), F(\mathfrak{x}, 1), \cdots, F(\mathfrak{x}, y)
$$

may be represented by a single number $w$ in the manner described above. Choosing the smallest possible value for $w$, we see that

$$
\left.F(\mathfrak{x}, y)=T_{y \mu w} w T_{0} w=A \mathfrak{x} \wedge \Lambda_{z}\left[z<y \rightarrow T_{S_{z}} w=B\left(\mathfrak{x}, z, T_{z} w\right)\right]\right\} .
$$

This may also be written in the form

$$
F(\mathfrak{x}, y)=T_{y} \mu w\left\{T_{0} w=A \mathfrak{x} \wedge \mu z\left\{T_{S_{z}} w \neq B\left(\mathfrak{x}, z, T_{z} w\right) \vee z=y\right\}=y\right\} .
$$

Thus we see that $F(x, y)$ has been obtained from the given functions $A$ and $B$, and the function $T$ by two applications of the $\mu$-rule. These are not in standard form, but can be reduced to standard form by elementary transformations, making use of the functions $x+y$, $x-y, x y$, and $0^{x}$. The function $T$ is obtained by substitution from $K, L, R$, and $S$. Thus to make possible the elimination of recursion, it is sufficient to adjoin $x+y, x-y, x y, 0^{x}, K, L$, and $R$ to the usual initial functions. All general recursive functions can then be obtained using substitution and the $\mu$-rule in the standard form.

We shall show that actually it is sufficient to adjoin $x+y$ and $Q$ to the initial functions.

We note first that $0^{x}=Q S S(Q x+Q S x)$. We shall next define a function $x^{1 / 2}$. This symbol, in distinction to $\left[x^{1 / 2}\right]$, will be used to de- 
note any function which gives the square root of $x$ when $x$ is a square, but whose values for other arguments are immaterial. A suitable definition is

$$
x^{1 / 2}=\mu y\{Q(x+2 y+1)=1\} .
$$

The condition in braces may also be written in the standard form $0^{Q S(x+y+y)}=0$.

It was proved by Euler that it is impossible to find four distinct squares forming an arithmetic progression. Using this fact, we may define $x^{2}$ as follows:

$$
\begin{aligned}
x^{2} & =\mu y\left\{Q y=1 \wedge Q\left(y+3 y^{1 / 2}+x+4\right)\right. \\
& =1 \wedge Q\left(y+2 y^{1 / 2}+2 x+4\right) \\
& \left.=1 \wedge Q\left(y+y^{1 / 2}+3 x+4\right)=1\right\} .
\end{aligned}
$$

In the first place, $y=x^{2}$ satisfies the condition. Conversely, if $y$ satisfies the condition, then $y$ is a square, so that $y^{1 / 2}$ indeed represents the square root of $y$. Thus $y+4 y^{1 / 2}+4$ is also a square so that the condition requires four squares in arithmetic progression. They must all be equal, hence $y^{1 / 2}=x$ or $y=x^{2}$. Thus $x^{2}$ is the only $y$ satisfying the condition in braces. The condition may be reduced to standard form by using

$$
a \neq 0 \wedge b \neq 0 \wedge c \neq 0 \wedge d \neq 0 \leftrightarrow 0^{a}+0^{b}+0^{c}+0^{d}=0 .
$$

The following definitions may now be made:

$$
\begin{aligned}
x-y & =\mu z\left\{Q\left((x+y)^{2}+x+3 y+1+z\right)=1\right\}, \\
{[x / 2] } & =\mu y\{S y+S y>x\}, \\
{\left[x^{1 / 2}\right] } & =\mu y\left\{(S y)^{2}>x\right\}, \\
x \cdot y & =\left[\left(\left((x+y)^{2}-x^{2}\right)-y^{2}\right) / 2\right], \\
{[x / y] } & =\mu z\{y \cdot S z>x \vee y=0\}, \\
R(x, y) & =x-y[x / y] .
\end{aligned}
$$

The conditions involving inequalities may be reduced to standard form using the difference function. Finally, $K$ and $L$ may also be obtained by substitution. Thus it is indeed sufficient to adjoin $x+y$ and $Q$ to the initial functions in order to obtain all general recursive functions by substitution and use of the $\mu$-rule.

We shall now prove that all general recursive functions can be obtained by substitution and inversion $A^{-1} x=\mu y\{A y=x\}$ if we adjoing $x+y$ and $E$ to the initial functions. The reduction of the general form of the $\mu$-rule to this form required use of the functions $J$, 
$K, L, x+y, x y$, and $0^{x}$. We needed $x+y$ and $Q$ in the general case. All of these functions can be obtained from

$$
x+y, \quad x-y, \quad x^{2}, \quad[x / 2], \quad\left[x^{1 / 2}\right], \quad 0^{x}
$$

by subtitution. Thus we need only to show that these functions can be obtained from the usual initial functions and $x+y$ and $E$ by substitution and inversion.

In the first place, we see that $E^{-1}(2 x)=x^{2}+2 x$. It follows easily that

$$
E\left(E^{-1}(2 x+2 y)+3 x+y+4\right)=x-y \quad \text { if } x \geqq y .
$$

Now we define

$$
x^{2}=E^{-1}(2 x)-2 x
$$

We may then put

$$
\operatorname{sgn} x=E S\left(x^{2}\right), \quad O^{x}=1-\operatorname{sgn} x .
$$

It is easily seen that the predecessor function $P$ can defined by

$$
P x=E_{\mu y}\{E S y=x\} \text {. }
$$

Now observing that $E S S E^{-1}$ is the characteristic function of even numbers, and that $E$ assumes all values for even arguments, we find that

$$
[x / 2]=E_{\mu y}\left\{2 E y+\operatorname{ESSE}^{-1} y=x\right\} \text {. }
$$

Clearly,

$$
x^{1 / 2}=[E P x / 2]+\operatorname{sgn} x
$$

if $x$ is a square, and

$$
\left[x^{1 / 2}\right]=(x-E x)^{1 / 2} .
$$

We have thus defined all the required functions.

Finally, we observe that instead of $E$ we may equally well adjoin either $K$ or $L$ to the initial functions (the sum $x+y$ being adjoined in any case). The pattern of values assumed by $K$ and $L$ is indicated in the following table:

$$
\begin{aligned}
x & =0,1,2,3,4,5,6,7,8,9, \cdots, \\
K x & =0,0,1,0,1,2,0,1,2,3, \cdots, \\
L x & =0,1,0,2,1,0,3,2,1,0, \cdots .
\end{aligned}
$$

In particular, $K x$ is the excess of $x$ over a triangular number. We 
notice that $K^{-1} x=x(x+3) / 2$. The following formulas are then easily verified:

$$
\begin{aligned}
& K\left(K^{-1}(x+y)+2 x+3\right)=x-y \quad \text { for } x \geqq y \text {, } \\
& x^{2}=2 K^{-1} x-3 x, \quad P x=K \mu y\{K S y=x\} \text {, } \\
& K x+L x=K P(x-K x)+K S(x-K x) \text {. }
\end{aligned}
$$

Thus $L x$ can be obtained by subtraction. Noting that of all representations of $x$ in the form $u^{2}+v$, the minimum value of $u+v$ is attained only when $u=\left[x^{1 / 2}\right]$ and $v=E x$, we see that $J(u, v)$ is smallest in this case. Hence

$$
E x=L \mu y\left\{(K y)^{2}+L y=x\right\} .
$$

Thus $K$ may be used as an initial function in place of $E$. To see that $L$ may equally well be used, we need only note the equation

$$
K x=L(x+2 L x+2) .
$$

4. General recursive functions of one variable. The results of this section, as far as Theorem 3, are exactly parallel to those of PRF, $\$ 7$. The omitted proofs can be copied from there practically verbatim.

It is easy to see that all general recursive functions of more than one variable can be obtained from general recursive functions of one variable, the function $x+y$, and the various identity functions, by substitution alone. Furthermore, as we shall show, all general recursive functions of one variable can be obtained without using functions of more variables in the process.

THEOREM 1. The functions of one variable which are obtainable from certain initial functions by substitution and inversion are also obtainable if substitution is not allowed except for defining functions of one variable.

THEOREM 2. If the initial functions include the identity and zero functions, and the function $x+y$, but no other function of more than one variable, then all functions of one variable obtainable by substitution and inversion are also obtainable by repeated use of the three formulas

$$
F x=A x+B x, \quad F x=B A x, \quad F x=B^{-1} x
$$

to define $F$ when $A$ and $B$ are known functions of one variable. The third formula is used only when $B$ assumes all values.

ThEOREM 3. All general recursive functions of one variable can be obtained by starting with the two functions $S$ and $E$, and repeatedly using any of the formulas 


$$
F x=A x+B x, \quad F x=B A x, \quad F x=B^{-1} x
$$

to construct a new function from the known functions $A$ and $B$. The third formula is used only when $B$ assumes all values.

Proof. The identity and zero functions of one variable, $I=I_{11}$ and $O=O_{1}$, can be defined by

$$
I x=E E^{-1} x, \quad O x=E S E^{-1}(x+x) .
$$

Thus by Theorem 2, we can obtain all the functions of one variable which we could get by substitution and inversion from all the identity and zero functions, $x+y, S$, and $E$. But by $\S 3$, all general recursive functions can be so obtained.

REMARK. In place of $E$ in this theorem, we may also use either $K$ or $L$. In virtue of the results of $\$ 3$, we need only note the definitions of $I$ and $O$,

$$
\begin{array}{ll}
I x=K K^{-1} x, & O x=K S K^{-1} x, \\
I x=L L^{-1} x, & O x=L\left(I x+L^{-1} x\right) .
\end{array}
$$

Furthermore, in place of $S$ we may also use $S O$. For without using $S$ we can define $I$, and then we put $S x=I x+S O x$.

REMARK. Comparing this theorem with the corresponding theorem of PRF, §7, we see that the only difference is the substitution of the scheme $F x=B^{-1} x$ for the scheme $F x=B^{x} O$, the result of this change being that we obtain all general recursive functions instead of just primitive recursive functions.

We shall now make a further reduction, eliminating the scheme $F x=A x+B x$ from the preceding theorem, a suitable change in the initial functions being made. It will be convenient to introduce the following notation. If $F x=A x+B x$ and $G x=C(A x, B x)$, we shall denote $F$ and $G$ by $A+B$ and $C(A, B)$.

THEOREM 4. All general recursive functions of one variable can be obtained by starting with a certain two primitive recursive functions and repeatedly using the formulas

$$
F x=B A x, \quad F x=B^{-1} x
$$

to construct a new function from known functions $A$ and $B$. The latter formula is used only when $B$ assumes all values.

Proof. We first make the observation that any finite set of initial functions can be replaced by two initial functions. Indeed, the functions $F_{1}, F_{2}, \cdots, F_{n}$ are evidently obtainable by substitution from the two functions, $K$ and 


$$
J\left(L, J\left(F_{1}, J\left(F_{2}, \cdots, J\left(F_{n-1}, F_{n}\right) \cdots\right)\right)\right) .
$$

Thus we need only show that a finite set of primitive recursive initial functions is possible.

To each general recursive function $F$, we make correspond another function $F^{*}$ by the formula

$$
F^{*}=J(F K, L),
$$

where $J, K$, and $L$ are the Cantor pairing functions. We know that $F$ can be generated from $S O$ and $K$ by addition, substitution, and inversion. Each step of this construction can be mirrored for the star functions in such a way that addition is unnecessary. Since

$$
K F^{*} J(I, I)=K J(F K, L) J(I, I)=K J(F, I)=F,
$$

we see that the function $F$ itself can then be recovered.

We notice first that

$$
B^{*} A^{*}=J(B K, L) J(A K, L)=J(B A K, L)=(B A)^{*} .
$$

If $B$ assumes all values, then $B^{*}$ does also, and we have

$$
\begin{aligned}
\left(B^{*}\right)^{-1} x & =\mu z\left\{B^{*} z=x\right\}=\mu z\{J(B K z, L z)=x\} \\
& =\mu z\{B K z=K x \wedge L z=L x\}=J(\mu y\{B y=K x\}, L x) \\
& =J\left(B^{-1} K x, L x\right)=\left(B^{-1}\right)^{*} x .
\end{aligned}
$$

We have used the fact that $J$ is a one-to-one monotone pairing function. Finally, we shall show that $(A+B)^{*}$ is obtained from $A^{*}$ and $B^{*}$ by substitution, making use of the special functions

$$
G_{1}=J(K, I), \quad G_{2}=J(K L, I) . \quad G_{3}=J(K+K L, L L L) .
$$

Indeed we have

$$
\begin{aligned}
A^{*} G_{1} & =J(A K, L) J(K, I)=J(A K, I), \\
B^{*} G_{2} & =J(B K, L) J(K L, I)=J(B K L, I), \\
G_{8} B^{*} G_{2} & =J(K+K L, L L L) J(B K L, I)=J(B K L+K, L L), \\
G_{3} B^{*} G_{2} A^{*} G_{1} & =J(B K L+K, L L) J(A K, I)=J(B K+A K, L),
\end{aligned}
$$

that is,

$$
(A+B)^{*}=G_{3} B^{*} G_{2} A^{*} G_{1} .
$$

Thus if $F$ is any general recursive function, the corresponding function $F^{*}$ can be obtained from the two initial functions $(S O)^{*}$ $=J(S O, L)$ and $K^{*}=J(K K, L)$ by use of substitution and inversion only, making use of the three auxiliary functions $G_{1}, G_{2}$, and $G_{3}$. 
Finally, $F$ itself is obtained by substitution from $F^{*}, K$, and $J(I, I)$. Thus all general recursive functions of one variable may be obtained by substitution and inversion from the seven functions,

$K, J(I, I), J(S O, L), J(K K, L), J(K, I), J(K L, I), J(K+K L, L L L)$.

As already remarked, these seven functions can be replaced by two suitable functions, so the proof of the theorem is complete.

We shall however investigate the possibility of replacing these seven functions by fewer functions of a simpler nature than one of the two obtained by the above process. We start by proving a lemma valid for arbitrary pairing functions.

Lemma. If $M$ and $N$ can be obtained by substitution from $I, K$, and $L$, then $J(M, N)$ can be obtained by substitution from $J(L K, K L)$ and $J(L, I)$.

Proof. We notice first that

$J(A K, B L) J(C x, D x)=J(A K J(C x, D x), B L J(C x, D x))=J(A C x, B D x)$.

Thus

$$
J(A K, B L) J(C, D)=J(A C, B D) .
$$

This and similar reductions will be used freely below. In particular, we observe the identities

$$
\begin{aligned}
J(L, K) J(A, B) & =J(B, A), \\
J(K K, L) J(A, B) & =J(K A, B), \\
J(L K, L) J(A, B) & =J(L A, B) .
\end{aligned}
$$

From these it is clear that all required functions $J(M, N)$ can be obtained by substitution from the four functions

$$
J(I, I), \quad J(L, K), \quad J(K K, L), \quad J(L K, L) .
$$

It remains to show that these four functions can in turn be obtained from the two functions

$$
G=J(L K, K L), \quad H=J(L, I) .
$$

The following computation shows that this is possible.

$$
\begin{aligned}
G H & =J(L K, K L) J(L, I)=J(L L, K), \\
G H^{2} & =J(L L, K) J(L, I)=J(L, L), \\
G H^{3} & =J(L, L) J(L, I)=J(I, I), \\
G^{2} H^{3} & =J(L K, K L) J(I, I)=J(L, K),
\end{aligned}
$$




$$
\begin{aligned}
G^{2} H^{4} & =J(L, K) J(L, I)=J(I, L), \\
G^{3} H^{4} & =J(L K, K L) J(I, L)=J(L, K L), \\
G^{3} H^{5} & =J(L, K L) J(L, I)=J(I, K), \\
G^{4} H^{5} & =J(L K, K L) J(I, K)=J(L, K K), \\
G^{2} H^{3} G^{4} H^{5} & =J(L, K) J(L, K K)=J(K K, L), \\
G H G^{2} H^{3} & =J(L L, K) J(L, K)=J(L K, L) .
\end{aligned}
$$

TheOREM 5. All general recursive functions of one variable can be obtained by substitution and inversion from the three functions $J(L K, K L)$, $J(I, S O), J(K+K L, I)$.

Proof. It is sufficient to show that we can define the seven functions listed at the end of the proof of Theorem 4. In the first place,

$$
\begin{gathered}
J(K+K L, I) J(I, S O)=J(I+K S O, J(I, S O))=J(I, J(I, S O)), \\
J(L K, K L) J(I, J(I, S O))=J(L, I) .
\end{gathered}
$$

Hence by the lemma we can obtain all functions $J(M, N)$ where $M$ and $N$ are obtained from $I, K, L$ by composition. Since the pairing function $J$ is assumed one-to-one, we obtain $K=J(K K, L K)$. The remaining functions are obtained in virtue of the equations

$$
\begin{aligned}
J(K+K L, L L L) & =J(K, L L L L) J(K+K L, I), \\
J(S O, L) & =J(L, L K) J(I, S O) .
\end{aligned}
$$

We shall now show that it is impossible to reduce the two initial functions in Theorem 4 to a single function of any sort.

THEOREM 6. There is no function $A$ from which all general recursive functions can be obtained by substitution and inversion.

Proof. Such a function $A$ would have to assume all values. For otherwise no function assuming all values could be obtained by substitution, and inversion would be impossible. If $A$ were a one-to-one mapping of the integers onto themselves, then only such permutations would be definable. Hence $A$ must assume some value more than once.

We shall show that the only functions assuming all values which are obtainable from $A$ are $A^{0}=I, A, A^{2}, A^{3}, \cdots$, and hence inversion is possible only for powers of $A$. Clearly, it is sufficient to show that

$$
F=A^{k}\left(A^{l}\right)^{-1} A^{m}\left(A^{n}\right)^{-1} \cdots A^{p}\left(A^{q}\right)^{-1}
$$

assumes all values only if it is a power of $A$. If $k<l$, then $F$ does not assume all values, since $A^{k}\left(A^{l}\right)^{-1}$ does not. Indeed, we know that 
$A^{l-k}$ assumes some value at least twice; hence if $A^{k}\left(A^{l}\right)^{-1}$ assumed all values, then $I=A^{l-k} A^{k}\left(A^{l}\right)^{-1}$ would have to assume some value twice. Hence $k \geqq l$, and then $A^{k}\left(A^{l}\right)^{-1}=A^{k-l} A^{l}\left(A^{l}\right)^{-1}=A^{k-l}$. Thus $F$ can be simplified. Repeating the argument, we find that if $F$ assumes all values, it must be a power of $\boldsymbol{A}$. Since no permutations are obtained except the identity, we do not obtain all general recursive functions.

5. Representation of general recursive functions in terms of primitive recursive functions. Starting from the Herbrand-Gödel metamathematical definition of general recursive function, Kleene showed that every such function can be expressed in the form

$$
F \mathfrak{x}=A \mu y\{B(\mathfrak{x}, y)=0\},
$$

where $A$ and $B$ are primitive recursive functions and the required $y$ exists for each $x$. Starting from the definition of this paper, we shall prove the possibility of such a representation, without using metamathematical arguments. We may also replace $\mu$ by $\iota$ in this formula.

THEOREM. Every general recursive function $F$ can be expressed in the form

$$
F \mathfrak{x}=A \iota y\{B(\mathfrak{x}, y)=0\}
$$

where $A$ and $B$ are primitive recursive functions, and for every $\mathfrak{x}$ there is $a$ unique $y$ such that $B(\mathfrak{x}, y)=0$.

Proof. By successive applications of the pairing functions, we can reduce the theorem to the special case $\mathfrak{x}=x$. We can then apply the results of $\$ 4$ concerning general recursive functions of one variable. We notice first that if $F$ is primitive recursive, the representation

$$
F x=F\llcorner y\{|x-y|=0\}
$$

may be used. Thus according to Theorem 4 of $\$ 4$ we need only check that the set of functions which can be represented in the form stated in the theorem is closed under substitution and inversion.

Suppose that

$$
F x=A \iota y\{B(x, y)=0\}, \quad G x=C \iota y\{D(x, y)=0\},
$$

where $A, B, C, D$ are primitive recursive. Then

$$
\begin{aligned}
F G x & =A \iota z\{B(G x, z)=0\} \\
& =A \iota z\{B(C \iota y\{D(x, y)=0\}, z)=0\} .
\end{aligned}
$$


In this formula, $y$ and $z$ are uniquely determined by $x$. Putting $w$ $=J(y, z)$ ( $J$ is taken to be Cantor's pairing function), we see that

$$
F G x=A L \imath w\{B(C K w, L w)=0 \wedge D(x, K w)=0\},
$$

which reduces to the required form. Thus the set of functions considered is closed under substitution.

Now consider inversion. Again suppose that

$$
F x=A \iota y\{B(x, y)=0\} .
$$

Let us assume first $B(x, y)=0$ determines $y$ as a monotone non-decreasing function of $x$. The inverse function is determined by the formula

$$
F^{-1} z=\mu x\{F x=z\}=\mu x\{A \iota y\{B(x, y)=0\}=z\} .
$$

Now $y$ is determined uniquely by $x$, and increases with $x$, hence the same is true of $w=J(x, y)$. Since the smallest possible $w$ corresponds to the smallest possible $x$, we see that

$$
F^{-1} z=K \mu w\{A L w=z \wedge B(K w, L w)=0\} .
$$

This can be reduced to the required form by the method of $\$ 2$.

If $B$ does not determine $y$ as a monotone function of $x$, we can find a new representation of $F$ with this property. Notice first that for a given $x$, we can choose $u$ so that $K u, K L u, \cdots, K L^{x} u$ all have preassigned values, and the smallest possible $u$ increases with each of them. Hence

$$
F x=A K L^{x_{\mu}} u\left\{B(0, K u)=0 \wedge B(1, K L u)=0 \wedge \cdots \wedge B\left(x, K L^{x} u\right)=0\right\},
$$

or

$$
F x=A K L^{x} \mu u\{G(x, u)=0\}
$$

where

$$
G(x, u)=\sum_{n=0}^{x} B\left(n, K L^{n} u\right) .
$$

Clearly, the smallest $u$ such that $G(x, u)=0$ increases with $x$, since more conditions are imposed on it. Putting $v=J\left(2^{x} u, L^{x} u\right)$, and noticing that we always have $J(2 z, 0) \geqq J(z, z)$, we see that $v$ also increases with $x$. It follows that

$$
F x=A K L \mu v\left\{G\left(x,\left[K v / 2^{x}\right]\right)=0 \wedge L v=L^{x}\left[K v / 2^{x}\right]\right\} .
$$

The functions occurring in the braces are all primitive recursive functions of $v$ and $x$, and the smallest $v$ satisfying the condition increases 
with $x$. We may now reduce to the desired form as in $\$ 2$.

Corollary. Any general recursive function of one variable can be expressed in the form $A B^{-1}$, where $A$ and $B$ are primitive recursive and $B$ assumes all values.

Proof. The reduction to this form is made as in $\$ 2$.

CoROllary. $A$ function $F \mathfrak{x}$ (of $n$ variables) is general recursive if and only if it has the form $F \mathfrak{x}=A C \mathfrak{x}$, where $A y$ and $0^{|y-C \mathfrak{x}|}$ are primitive recursive functions (of one and $n+1$ variables, respectively). ${ }^{5}$

Proof. If $F$ is general recursive, it has the form

$$
F \mathfrak{x}=A \iota y\{B(\mathfrak{x}, y)=0\},
$$

where $A$ and $B$ are primitive recursive. Putting

$$
C \mathfrak{x}=\iota \mathfrak{y}\{B(\mathfrak{x}, y)=0\},
$$

we see that

$$
0^{\left|y-c_{x}\right|}=0^{B(y, y)}
$$

is primitive recursive. Conversely, suppose $F \mathfrak{x}=A C \mathfrak{x}$, where $A y$ and $0^{|y-c \gamma|}$ are primitive recursive. Then

$$
F \mathfrak{r}=A \iota y\left\{0^{\left|y-C_{\mathfrak{z}}\right|}=1\right\}
$$

is general recursive.

Berkeley, Calif.

- Stated by A. Tarski in a seminar at the University of California in the summer of 1943. Also see Th. Skolem, Some remarks on recursive arithmetic, Norske Videnskabers Selskabs, Forhandlinger vol. 17 (1944) pp. 103-106. 